\title{
An Axiomatic Derivation of the Logarithmic Function as a Cardinal Utility Function on Money Income Levels
}

\author{
Mitsunobu Miyake \\ Graduate School of Economics and Management, Tohoku University, Sendai, Japan \\ Email: miyake@econ.tohoku.ac.jp
}

Received November 27, 2013; revised December 27, 2013; accepted January 4, 2014

Copyright (C) 2014 Mitsunobu Miyake. This is an open access article distributed under the Creative Commons Attribution License, which permits unrestricted use, distribution, and reproduction in any medium, provided the original work is properly cited. In accordance of the Creative Commons Attribution License all Copyrights @ 2014 are reserved for SCIRP and the owner of the intellectual property Mitsunobu Miyake. All Copyright (c) 2014 are guarded by law and by SCIRP as a guardian.

\section{ABSTRACT}

This note elaborates Suppes' (1977, Erkenntnis Vol. 11, No. 1, pp 233-250) derivation of the logarithmic function as a consumer's cardinal utility function on money income levels, in which the consumer's preferences are specified by a level comparison relation and a difference comparison relation. Without assuming Suppes' hypothesis (Bernoulli's hypothesis or Weber-Fechner law), which asserts that the utility values are proportional to the logarithmic values of income levels, it is shown that the representability of the two relations by logarithmic utility function can be characterized only by the three (mutually independent) axioms on the relations.

\section{KEYWORDS}

Logarithmic Utility Function; Suppes’ Hypothesis; Bernoulli’s Hypothesis; Weber-Fechner Law; Level Comparison; Difference Comparison; Monotonicity Axiom; Consistency Axiom; Homogeneity Axiom

\section{Introduction}

The logarithmic utility function on money income levels is widely adapted as a utility function representing a consumer's preferences on income levels in the models of the welfare economics and the growth theory ${ }^{1}$. Specifically, the logarithmic utility function is used as a typical utility function representing the law of diminishing marginal utilities, which is a key property of utility functions in the models leading some equity-regarding prescriptions such as the progressive income tax schedule. In the neoclassical (Paretian) utility theory, a consumer's preferences on income levels is specified by a level comparison relation and a level comparison relation is re-presented by the logarithmic utility function if and only if the relation satisfies the monotonicity axiom, i.e., the larger levels of income are more desirable. However, the relation is represented by the logarithmic function as an

\footnotetext{
${ }^{1}$ Watts [1] introduces a poverty index, which can be interpreted as the differences of the logarithmic utility values. See [2,3] for the Watts index. For the examples in the growth theory, see [4] Propositions 4 and 5, [5] Chapter 6 Example 6.4, and [6] Appendix Figure A2-2.
}

ordinal utility function ${ }^{2}$. Consequently, the equilibria or solutions of the specific economic models depend on the selections of the utility representations, if the definitions of the equilibria or solutions involve the cardinal properties of the utility functions. For example, the Nash bargaining solution is not well-defined, if the utility representations are determined unique up to monotone transformations.

Suppes [7] Section 2 derives the logarithmic utility function based on a cardinal utility representation theorem ([7] Theorem 2) for a general class of preferences including non-monotonic preferences, in which individual preferences are specified by the difference comparison relation as well as the level comparison relation. Concretely, Suppes shows that the utility representation is determined unique up to positive affine transformations,

\footnotetext{
${ }^{2}$ Namely the following three statements for a (rational) level comparison relation $R$ on positive income levels are mutually equivalent: 1 ) the relation $R$ satisfies the monotonicity axiom, i.e., $x>y \Rightarrow x R y$ and not $y R x$; 2) the relation $R$ is represented by the logarithmic function, i.e., $\quad x R y \Leftrightarrow \log x \geq \log y$; 3) the relation $R$ is represented by any function in $\mathrm{M}$ and no function in $\mathrm{M}^{\mathrm{C}}$ represents $R$, where $\mathrm{M}$ is the set of all increasing functions on the income levels.
} 
which means that the derived utility function is a cardinal utility function representing the two relations simultaneously, and then the equilibria or solutions involving the cardinal properties are well-defined under the utility representation. However, Suppes ([7] Section II Hypothesis $\mathrm{U}$ ) assumes that the utility values are proportional to the logarithmic values of income levels to determine the logarithmic utility function among the general class of utility functions ${ }^{3}$.

In this paper, without assuming such a superficial hypothesis on the derived utility values, it is shown that the representability of the two relations by logarithmic function can be characterized only by the axioms on the relations. Specifically, a pair of the relations on the alternatives is called a preference structure, and it is shown that a preference structure is represented by the logarithmic function as a cardinal utility function if and only if the preference structure satisfies the (mutually independent) three axioms: monotonicity, consistency and homogeneity axioms. The homogeneity axiom is the new axiom introduced by this paper and the monotonicity and consistency axioms are standard axioms in the cardinal utility theory based on the difference comparisons.

\section{The Model and Results}

We introduce some fundamental concepts and definitions used in the cardinal utility theory based on the difference comparisons and show the characterization result for the logarithmic utility function ${ }^{4}$.

\subsection{The Model}

The set of all possible levels of money income is the set of positive real numbers denoted by $\mathrm{X} \equiv \mathbf{R}_{++}$. A level comparison relation $R$ is a complete and transitive binary relation on $\mathrm{X}$. The expression $x R y$ means that $x$ is preferred to $y$. The symmetric and asymmetric parts of $R$ are denoted by $I$ and $S$, respectively. A difference comparison relation $R^{*}$ on $\mathrm{X}$ is a complete and transitive quaternary relation on $\mathrm{X}$. The expression $(x \rightarrow y) R^{*}(z \rightarrow w)$ means that the transition (path) from $x$ to $y$ is preferred to the transition from $z$ to $w$. We assume that all the possible transitions $(x \rightarrow y)$ are completed for a fixed length of time interval and the underlying price vector is fixed over the time interval; otherwise, the money income levels are

\footnotetext{
${ }^{3}$ In the literature of psychophysics, almost the same hypothesis called Weber-Fechner law is assumed for deriving a logarithmic function to represent the relationship between the stimuli and sensations. For the Weber-Fechner law, see [8]. In case of the expected utility function, which is also determined unique up to positive affine transformations, almost the same hypothesis stated in a differential form is assumed by Bernoulli to derive the logarithmic utility function. For the derivation of logarithmic utility function based on Bernoulli hypothesis, see [9] Section 3 and [10] Historical Note 6 Classic logarithmic utility.

${ }^{4}$ For the cardinal utility theory based on the difference comparisons, see [11-13].
}

assumed to be adjusted suitably (PPP adjustment). The symmetric and asymmetric parts of $R^{*}$ are denoted by $I^{*}$ and $S^{*}$, respectively. A preference structure on $\mathrm{X}$ is a pair of a level comparison relation $R$ and a difference comparison relation $R^{*}$ on $\mathrm{X}$. The preference structure on $\mathrm{X}$ is denoted by $\left(R, R^{*}\right)$. A preference structure $\left(R, R^{*}\right)$ on $\mathrm{X}$ is represented by a real-valued function $u$ on $\mathrm{X}$ if and only if the following two assertions hold:

1) $x R y \Leftrightarrow u(x) \geq u(y)$ for all $x, y \in \mathrm{X}$,

2) $(x \rightarrow y) R^{*}(z \rightarrow w) \Leftrightarrow u(y)-u(x) \geq u(w)-u(z)$ for all $x, y, z, w \in \mathrm{X}$. This definition implies that, for a given real-valued function $u$ on $\mathrm{X}$, there uniquely exists a preference structure $\left(R, R^{*}\right)$ which is represented by the function $u$, i.e., if $\left(R_{a}, R_{a}^{*}\right)$ and $\left(R_{b}, R_{b}^{*}\right)$ are represented by $u$, then $\left(R_{a}, R_{a}^{*}\right)^{a}=\left(R_{b}, R_{b}^{*}\right)$. Specifically, let $\left(R_{0}, R_{0}^{*}\right)$ be the preference structure represented by the logarithmic function, i.e.,

3) $x R_{0} y \Leftrightarrow \log x \geq \log y$ for all $x, y \in \mathrm{X}$,

4) $(x \rightarrow y) R_{0}^{*}(z \rightarrow w) \Leftrightarrow \log y-\log x \geq \log w-\log z$ for all $x, y, z, w \in \mathrm{X}$. Since the logarithmic function is monotone, the condition 3) means that $R_{0}$ is the monotone level comparison relation. For $R_{0}^{*}$, we need a lemma proved in Appendix B:

Lemma $1 \log y-\log x \geq \log w-\log z \Leftrightarrow(y / x) R(w / z) \Leftrightarrow$ $(y-x) / x \geq(w-z) / z$ for all $x, y, z, w \in X$.

It follows from Lemma 1 that the condition 4) means that the difference comparison relation $R_{0}^{*}$ is determined by the simple rule that the differences with larger growth rates are more desirable, i.e.,

$$
(x \rightarrow y) R_{0}^{*}(z \rightarrow w) \Leftrightarrow(y-x) / x \geq(w-z) / z .
$$

\subsection{Results}

We introduce the following axioms to characterize the representability of a preference structure by the logarithmic function:

Monotonicity $x>y \Rightarrow x S y$ for all $x, y \in \mathrm{X}$.

Consistency There exists some $z \in X$ such that $(z \rightarrow x) R^{*}(z \rightarrow y) \Leftrightarrow x R y$ for all $x, y \in \mathrm{X}$.

Homogeneity $(x \rightarrow y) I^{*}(t x \rightarrow t y)$ for all $x, y \in \mathrm{X}$ and all $t>0$.

The monotonicity axiom means that the larger levels of income are more desirable, and the consistency axiom means that there exists some $z \in X$ such that the difference comparison on $(z \rightarrow x)$ and $(z \rightarrow y)$ coincides with the level comparison on $x$ and $y$ for all $x, y \in \mathrm{X}$. The homogeneity axiom means that two transitions are indifferent if one transition is given by multiplying a positive number both for the initial and ending levels of another transition. Then we have the following proposition:

Proposition Let $\left(R, R^{*}\right)$ be a preference structure on $\mathrm{X}$ and let $\mathrm{L}$ be a set of real-valued functions $f$ on $\mathrm{X}$ defined by $\mathrm{L}=\{$ f: there exist $a>0$ and $b$ such that 
$f(x)=a \cdot \log x+b$ for all $x \in \mathrm{X}\}$. Then the following three statements are mutually equivalent:

1) $\left(R, R^{*}\right)$ satisfies the monotonicity, consistency and homogeneity axioms;

2) $\left(R, R^{*}\right)$ coincides with $\left(R_{0}, R_{0}^{*}\right)$, i.e., $\left(R, R^{*}\right)$ is represented by the logarithmic function;

3) $\left(R, R^{*}\right)$ is represented by any function in $\mathrm{L}$ and no function in $\mathrm{L}^{\mathrm{C}}$ represents $\left(R, R^{*}\right)$.

Proposition is proved in Appendix A. The three axioms in the assertion 1) of Proposition are mutually independent, which can be proved by constructing the counter examples: Define a preference structure $\left(R_{1}, R_{1}^{*}\right)$ by

$$
x I_{1} y \text { and }(x \rightarrow y) I_{1}^{*}(z \rightarrow w)
$$

for all $x, y, z, w \in \mathrm{X}$. The preference structure $\left(R_{1}, R_{1}^{*}\right)$ satisfies the homogeneity and consistency axioms, but it does not satisfy the monotonicity axiom, which implies that the monotonicity axiom is independent. Let $R_{2}^{*}$ be a difference comparison relation defined by

$$
(x \rightarrow y) R_{2}^{*}(z \rightarrow w) \Leftrightarrow(y-x) / x \leq(w-z) / z
$$

for all $x, y, z, w \in \mathrm{X}$, and let $R_{3}^{*}$ be a difference comparison relation defined by

$$
(x \rightarrow y) R_{3}^{*}(z \rightarrow w) \Leftrightarrow(y-x) \geq(w-z)
$$

for all $x, y, z, w \in \mathrm{X}$. Letting $R_{0}$ be the monotonic level comparison relation again, the preference structure $\left(R_{0}, R_{2}^{*}\right)$ satisfies the monotonicity and homogeneity axioms, but it does not satisfy the consistency axiom, which implies that the consistency axiom is independent. In fact, it holds that $2 S_{0} 1$ and $(z \rightarrow 1) S_{2}^{*}(z \rightarrow 2)$ for all $z \in \mathrm{X}$. The preference structure $\left(R_{0}, R_{3}^{*}\right)$ satisfies the monotonicity and consistency axioms, but it does not satisfy the homogeneity axiom, which implies that the homogeneity axiom is independent.

\section{Conclusions}

A consumer's preferences over money income levels are specified by a level comparison relation and a difference comparison relation. A pair of the relations is called a preference structure and it is shown that a preference structure is cardinally represented by the logarithmic function if and only if the preference structure satisfies the three mutually independent axioms on the preference structures. This result clarifies the mutually independent axioms characterizing the preference structure represented by the logarithmic function as a cardinal utility function. In particular, this result enables us to interpret the per capita national income measured by the logarithmic scale as the utility level of the income, and axiomatically cha- racterizes the simple rule evaluating the differences of per capita national income levels based on their growth rates under the monotonic level comparison relation.

\section{Funding}

The research for this paper is partially supported by Ministry of Education, Science and Culture through JSPS Grant-in-Aids for Scientific Research No. 24530191.

\section{REFERENCES}

[1] H. Watts, “An Economic Definition of Poverty,” In: D. P. Moynihan, Ed., On Understanding Poverty, Basic Books, New York, Chapter 11, 1968, pp. 316-329.

[2] S. R. Chakravarty, “A New Index of Poverty,” Mathematical Social Sciences, Vol. 6, No. 3, 1983, pp. 307-313. http://dx.doi.org/10.1016/0165-4896(83)90064-1

[3] B. Zheng, "An Axiomatic Characterization of the Watts Poverty Index,” Economics Letters, Vol. 42, No. 1, 1993, pp. 81-88. http://dx.doi.org/10.1016/0165-1765(93)90177-E

[4] E. Farhi and I. Werning, "Inequality and Social Discounting,” Journal of Political Economy, Vol. 115, No. 3, 2007, pp. 365-402. http://dx.doi.org/10.1086/518741

[5] D. Acemoglu, "Introduction to Modern Economic Growth," Princeton University Press, Princeton, 2008.

[6] O. Blanchard, "Macroeconomics," 5th Edition, Prentice Hall, Upper Saddle River, 2009.

[7] P. Suppes, “The Distributive Justice of Income Inequality," Erkenntnis, Vol. 11, No. 1, 1977, pp. 233-250. http://dx.doi.org/10.1007/BF00169854

[8] D. J. Murray, "A Perspective for Viewing the History of Psychophysics,” Behavioral and Brain Sciences, Vol. 16, No. 1, 1993, pp. 115-115. http://dx.doi.org/10.1017/S0140525X00029277

[9] H. Dalton, "The Measurement of the Inequality of Incomes,” Economic Journal, Vol. 30, No. 119, 1920, pp. 348-361. http://dx.doi.org/10.2307/2223525

[10] P. A. Samuelson, "St. Petersburg Paradoxes: Defanged, Dissected, and Historically Described," Journal of Economic Literature, Vol. 15, No. 1, 1977, pp. 24-55.

[11] F. Alt, "Über die Mebarkeit des Nutzens," Zeitschrift für Nationalökonomie, Vol. 7, No. 2, 1936, pp. 161-169. http://dx.doi.org/10.1007/BF01316465

[12] L. S. Shapley, "Cardinal Utility Comparisons from Intensity Comparisons,” Report R-1683-PR, The Rand Corporation, Santa Monica, 1975.

[13] V. Köberling, "Strength of Preference and Cardinal Utility," Economic Theory, Vol. 27, No. 2, 2006, pp. 375-391. http://dx.doi.org/10.1007/s00199-005-0598-5

[14] H. L. Royden, "Real Analysis,” 3rd Edition, Macmillan, New York, 1988. 


\section{Appendix A}

Proof of Proposition: We can easily show that the assertion 3) implies the assertion 2) and that the assertion 2) implies the assertion 1). First, we show that the assertion 1) implies the assertion 2). Suppose that $\left(R, R^{*}\right)$ satisfies the three axioms. By the contraposition of the monotonicity axiom that

$$
x R y \Rightarrow x \geq y \text { for all } x, y \in \mathrm{X} .
$$

Since $R$ is a complete and transitive binary relation on $\mathrm{X}$, it holds that

$$
x=y \Rightarrow x I y \text { for all } x, y \in \mathrm{X} .
$$

It holds by the monotonicity axiom and (2) that

$$
x \geq y \Rightarrow x R y \text { for all } x, y \in \mathrm{X} .
$$

Hence it holds by (1) and (3) that

$$
x \geq y \Leftrightarrow x R y \text { for all } x, y \in \mathrm{X} .
$$

Since $\log (\cdot)$ is increasing on $X$, it holds by (4) that

$$
\log x \geq \log y \Leftrightarrow x R y \quad \text { for all } x, y \in X \text {. }
$$

Let $s$ be an element in X satisfying the condition in the consistency axiom, i.e.,

$$
x R y \Leftrightarrow(s \rightarrow x) R^{*}(s \rightarrow y) \text { for all } x, y \in \mathrm{X} .
$$

It holds by the homogeneity axiom that

$$
(x \rightarrow y) I^{*}(s \rightarrow(s y / x)) \text { and }(z \rightarrow w) I^{*}(s \rightarrow(s w / z)) \text {, }
$$

which implies that

$$
\begin{aligned}
& (x \rightarrow y) I^{*}(z \rightarrow w) \\
& \Leftrightarrow(s \rightarrow(s y / x)) I^{*}(s \rightarrow(s w / z)) .
\end{aligned}
$$

We have by (5) that

$$
(s \rightarrow(s y / x)) R^{*}(s \rightarrow(s w / z)) \Leftrightarrow(s y / x) R(s w / z) .
$$

Moreover, we have by (4) that

$$
\begin{aligned}
& (s y / x) R(s w / z) \Leftrightarrow(y / x) R(w / z) \\
& \Leftrightarrow(y-x) / x \geq(w-z) / z .
\end{aligned}
$$

Hence it holds by (6), (7) and (8) that

$$
\begin{aligned}
& (x \rightarrow y) R^{*}(z \rightarrow w) \\
& \Leftrightarrow(y-x) / x \geq(w-z) / z \text { for all } x, y, z, w \in X .
\end{aligned}
$$

It holds by (9) and Lemma 1 that

$$
(x \rightarrow y) R^{*}(z \rightarrow w) \Leftrightarrow \log y-\log x \geq \log w-\log z
$$

for all $x, y, z, w \in X$. Thus the assertion 2) holds.

Second, we will show that the assertion 2) implies the assertion 3). Suppose that the assertion 2) holds, i.e., $\left(R, R^{*}\right)=\left(R_{0}, R_{0}^{*}\right)$. Since the assertion 2) implies the assertion 1), $\left(R_{0}, R_{0}^{*}\right)$ satisfies the three axioms. Moreover, we can easily show that $\left(R_{0}, R_{0}^{*}\right)$ is represented by any function in $\mathrm{L}$. There remains to show that no function in $\mathrm{L}^{\mathrm{C}}$ represents $\left(R_{0}, R_{0}^{*}\right)$. Suppose that a real-valued function $g$ on $\mathrm{X}$ represents $\left(R_{0}, R_{0}^{*}\right)$, i.e., it holds that

$$
\begin{aligned}
& x R_{0} y \Leftrightarrow g(x) \geq g(y) \text { and } \\
& (x \rightarrow y) R_{0}^{*}(z \rightarrow w) \Leftrightarrow g(y)-g(x) \geq g(w)-g(z) \\
& \text { for all } x, y, z, w \in \mathrm{X}
\end{aligned}
$$

Define a function $f: \mathbf{R} \rightarrow \mathbf{R}$ by $f(t)=g\left(e^{t}\right)$ for all $t \in \mathbf{R}$. Then it holds that

$$
f(\log x)=g\left(e^{\log x}\right)=g(x) \text { for all } x \in X .
$$

We need a lemma proved in Appendix B:

\section{Lemma 2: 1)}

$$
\beta-\alpha=\delta-\gamma \Leftrightarrow f(\beta)-f(\alpha)=f(\delta)-f(\gamma)
$$

for all $\alpha, \beta, \gamma, \delta \in \mathbf{R}$. 2) $f(\cdot)$ is continuous and increasing on $\mathbf{R}$.

Setting $a^{*}=f(1)-f(0)>0$ and $b^{*}=f(0)$, we will prove that $f(t)=a^{*} t+b^{*}$ for all $t \in \mathbf{R}$. Suppose that $t$ is a rational number, i.e., there exists a pair of integers $(p, q)$ such that

$$
t=q / p, q \geq 0 \text { and } p \neq 0 .
$$

Using the induction arguments with respect to $q=0,1,2, \cdots$ for a fixed $p \neq 0$, it holds by Lemma 21 ) that

$$
\begin{gathered}
f(q / p)=[f(1 / p)-f(0)] q+f(0) \\
\text { for all integers } q \geq 0 \text { and } p \neq 0
\end{gathered}
$$

Case $1(t \geq 0)$ Setting $q=p$ in (13), we have that

$$
\begin{aligned}
& f(1)=[f(1 / p)-f(0)] p+f(0) \text { and } \\
& f(1 / p)=[f(1)-f(0)] / p+f(0) .
\end{aligned}
$$

Hence, we have by (13) and this that

$$
f(q / p)=(q / p)[f(1)-f(0)]+f(0) .
$$

Since $a^{*}=f(1)-f(0)$ and $b^{*}=f(0)$, we have that

$$
f(t)=a^{*} t+b^{*} \text { for all rational numbers } t \geq 0 \text {. }
$$

Since $f(\cdot)$ is continuous on $\mathbf{R}$ by Lemma 22 ), we have that

$$
f(t)=a^{*} t+b^{*} \text { for all real numbers } t \geq 0 .
$$

Case $2(t<0)$ It holds by (12) that $p<0$. Setting $q=-$ $p>0$ in (13), we have that

$$
\begin{aligned}
& f(-1)=-[f(1 / p)-f(0)] p+f(0) \text { and } \\
& f(1 / p)=[f(0)-f(-1)] / p+f(0) .
\end{aligned}
$$


Hence we have by (13) and this that

$$
f(q / p)=(q / p)[f(0)-f(-1)]+f(0) .
$$

Since $f(1)-f(0)=f(0)-f(-1)$ by Lemma 21 ), we have that $a^{*}=f(0)-f(-1)>0$. Hence we have that

$$
f(t)=a^{*} t+b^{*} \text { for all rational numbers } t<0 \text {. }
$$

Since $f(\cdot)$ is continuous on $\mathbf{R}$ by Lemma 22 ), we have that

$$
f(t)=a^{*} t+b^{*} \text { for all real numbers } t<0 \text {. }
$$

Thus it holds by (11) that $g(\cdot)=a^{*} \log (\cdot)+b^{*} \in \mathrm{L}$ and that no function in $L^{C}$ represents $\left(R_{0}, R_{0}^{*}\right)$.

\section{Appendix B}

Proof of Lemma 1 It holds that

$$
\begin{gathered}
\log y-\log x \geq \log w-\log z \\
\Leftrightarrow \log (y / x) \geq \log (w / z) \\
\Leftrightarrow(y / x) \geq(w / z) \\
\Leftrightarrow(y / x)-(x / x) \geq(w / z)-(z / z) \\
\Leftrightarrow(y-x) / x \geq(w-z) / z
\end{gathered}
$$

for all $x, y, z, w \in X$.

Proof of Lemma 21 ) Since $\left(R_{0}, R_{0}^{*}\right)$ satisfies the three axioms, it holds by Lemma 1 and (10) that

$$
\begin{aligned}
& y / x=w / z \\
& \Leftrightarrow(y-x) / x=(w-z) / z \\
& \Leftrightarrow(x \rightarrow y) I_{0}^{*}(z \rightarrow w) \\
& \Leftrightarrow g(y)-g(x)=g(w)-g(z)
\end{aligned}
$$

For any $\alpha, \beta, \gamma, \delta \in \mathbf{R}$, set $x^{*}=e^{\alpha}, y^{*}=e^{\beta}, z^{*}=e^{\gamma}$ and $w^{*}=e^{\delta}$. Then we have by (14) that

$$
\begin{aligned}
& y^{*} / x^{*}=w^{*} / z^{*} \\
& \Leftrightarrow g\left(y^{*}\right)-g\left(x^{*}\right)=g\left(w^{*}\right)-g\left(z^{*}\right) .
\end{aligned}
$$

It holds by this and (11) that

$$
\begin{aligned}
& \beta-\alpha=\delta-\gamma \Leftrightarrow y^{*} / x^{*}=w^{*} / z^{*} \\
& \Leftrightarrow g\left(y^{*}\right)-g\left(x^{*}\right)=g\left(w^{*}\right)-g\left(z^{*}\right) \\
& \Leftrightarrow f(\beta)-f(\alpha)=f(\delta)-g(\gamma) .
\end{aligned}
$$

2) Since $g(x)$ is (strictly) increasing on $X$ by (10), and since $e^{t}$ is (strictly) increasing on $\mathbf{R}, f(t) \equiv g\left(e^{t}\right)$ is (strictly) increasing on $\mathbf{R}$. Hence it holds by [14] Chapter 5 Theorem 3 p.100 that there are at most countable number of points at which $f$ is not continuous. Thus there is a point $\alpha$ in $\mathbf{R}$ at which $f$ is continuous. Let $\beta$ be a point in $\mathbf{R}$, and let $\left\{\beta_{m}\right\}$ be a sequence in $\mathbf{R}$ converging to $\beta$. Define a sequence $\left\{\alpha_{m}\right\}$ in $\mathbf{R}$ by

$$
\alpha_{m}=\alpha-\beta+\beta_{m} \text { for all } m=1,2, \cdots \text {. }
$$

Hence we have Lemma 2 1) that

$$
f\left(\alpha_{m}\right)-f(\alpha)=f\left(\beta_{m}\right)-f(\beta) \text { for all } m=1,2, \cdots .
$$

Since $\lim \alpha_{m}=\alpha$ and $f$ is continuous at $\alpha$, we have that $\lim f\left(\beta_{m}\right)=f(\beta)$. 